\title{
Estimating Average Variation About the Population Mean Using Geometric Measure of Variation
}

\author{
Troon John Benedict ${ }^{1}$, Karanjah Anthony ${ }^{2}$, Alilah Anekeya David ${ }^{3}$ \\ ${ }^{1}$ Department of Mathematics and Physical Science, Maasai Mara University, Narok, Kenya \\ ${ }^{2}$ Department of Mathematic, Multimedia University, Nairobi, Kenya \\ ${ }^{3}$ Department of Mathematics and Statistics, Masinde Muliro University of Science and Technology, Kakamega, Kenya
}

Email address:

troonbenedict@gmail.com (T. J. Benedict), karanjah@mmarau.ac.ke (K. Anthony), aliladavid2010@gmail.com (A. A. David)

\section{To cite this article:}

Troon John Benedict, Karanjah Anthony, Alilah Anekeya David. Estimating Average Variation About the Population Mean Using Geometric Measure of Variation. International Journal of Statistical Distributions and Applications. Vol. 6, No. 2, 2020, pp. 23-35.

doi: $10.11648 /$ j.ijsd.20200602.11

Received: September 17, 2019; Accepted: October 16, 2019; Published: August 25, 2020

\begin{abstract}
Measures of dispersion are important statistical tool used to illustrate the distribution of datasets. These measures have allowed researchers to define the distribution of various datasets especially the measures of dispersion from the mean. Researchers and mathematicians have been able to develop measures of dispersion from the mean such as mean deviation, variance and standard deviation. However, these measures have been determined not to be perfect, for example, variance give average of squared deviation which differ in unit of measurement as the initial dataset, mean deviation gives bigger average deviation than the actual average deviation because it violates the algebraic laws governing absolute numbers, while standard deviation is affected by outliers and skewed datasets. As a result, there was a need to develop a more efficient measure of variation from the mean that would overcome these weaknesses. The aim of the paper was to estimate the average variation about the population mean using geometric measure of variation. The study was able to use the geometric measure of variation to estimate the average variation about the population mean for un-weighted datasets, weighted datasets, probability mass and probability density functions with finite intervals, however, the function faces serious integration problems when estimating the average deviation for probability density functions as a result of complexity in the integrations by parts involved and also integration on infinite intervals. Despite the challenge on probability density functions, the study was able to establish that the geometric measure of variation was able to overcome the challenges faced by the existing measures of variation about the population mean.
\end{abstract}

Keywords: Standard Deviation, Geometric Measure of Variation, Deviation About the Mean, Average, Mean, Absolute Deviation, Estimation

\section{Introduction}

Geometric measure of variation about the mean, is a new measure of variation about the mean, that intends to solve the weaknesses of the current measures of variation about the mean, by using geometric averaging, to estimate the average deviation about the mean. Geometric averaging is a suitable measure of variation because based on past research it is not affected by outliers and skewed datasets. The technique also average product of numbers and not sum, a factor which makes it not to violate the algebraic laws [4, 19, 21].

Currently, there are three known measures of variation about the mean; Mean deviation which is the average absolute deviation about the mean, variance which is the average of squared deviations about the mean and standard deviation which is the square-root of average squared deviation about the mean [17]. Past studies have established that the existing measures of variation about the mean are not $100 \%$ efficient, this is due to various issue that arises during their use in estimating the average variation about the mean $[2,3,11-13,16,18,20]$. For example, mean deviation has been determined by past studies has to violate the algebraic number theory. Based on the algebraic number theory, given that the mean deviation is an average of absolute deviations, an absolute number on the field $\mathrm{P}$ such that $|\bullet|: p \rightarrow \mathfrak{R}^{>0}$ must satisfy 
the following conditions [5];

1. $|a|=0$ iff $a=0 \quad \forall a \in p$

2. $|a b|=|a||b| \forall a, b \in p$

3. $|a+b|<|a|+|b| \forall a, b \in p$

The mean deviation about the mean is given by the function [17];

$$
M A D=\frac{\sum_{i=1}^{n}\left|v_{i}-\bar{v}\right|}{n}
$$

where $\left|v_{i}-\bar{v}\right|$ is the absolute deviation from the mean

This measure of deviation from the mean as an absolute number, violates the algebraic laws as illustrated by the third condition (3), hence the average deviation about the mean estimated by the measure are not accurate because;

$$
\frac{\sum_{i=1}^{n}\left|v_{i}-\bar{v}\right|}{n}>\frac{\sum_{i=1}^{n}\left(v_{i}-\bar{v}\right)}{n}
$$

Therefore, the measure always gives bigger estimates than the actual deviation about the mean. However, the measure argues on the basis of the theory behind measurement of average deviation about the mean, which assumes that for measuring of deviation from the mean, the metric (the distance from the mean) is more important than the sign of the deviation. Mean deviation has also been determined by past studies not to allow further algebraic application because of the absolution, as a result the measure is not considered as efficient [6].

A second measure of variation about the mean is variance which is given by the function $[1,17]$;

$$
\operatorname{var}=\frac{\sum_{i=1}^{n}\left(v_{i}-\bar{v}\right)^{2}}{n}
$$

Where $\left(v_{i}-\bar{v}\right)^{2}$ is the squared deviation from the mean

This measure of variation from the mean allows for further algebraic manipulations which is an improvement from the mean deviation, it also do not violate the algebraic number theory, however, the average of deviation about the mean given by the measure are squared, hence, are not of the same unit as the initial datasets (squared). This makes the results given by the formula to be inappropriate $[1,17]$.

The last measure of variation about the mean is standard deviation, which is an improvement on variance by giving results which are of the same units as initial datasets. The measure is usually estimated by $[1,3,13,16]$;

$$
S D=\sqrt{\frac{\sum_{i=1}^{n}\left(v_{i}-\bar{v}\right)^{2}}{n}}
$$

Over the years, standard deviation has been the most widely used measure of variation about the mean, because it is a capable of further algebraic manipulation and it also solves the problem of variance by giving estimates which are of the same unit as the original datasets (square-root). However, past studies have determined that standard deviation is affected by outliers and skewed datasets, factors which makes this measure not to be efficient especially when dealing with datasets which have outliers and those that are skewed $[2-3,11-13,16,18,20]$.

Given the shortcomings of the three existing measures of variation about the mean. The paper aimed at a estimate the average variation about the population mean using the geometric measure of variation, which is would overcome the weakness of the current measures of variation about the mean by not violating the algebraic laws, giving estimates which are of the same unit as the initial datasets, not affected by outliers and skewed datasets, and allows further algebraic manipulations to be carried on it. This is because, geometric measure of variation would use geometric averaging, an averaging technique that average products hence not violating the algebraic laws behind absolute number. And also, an averaging technique that has been determined not to be affected by outliers and skewed datasets.

\section{Method}

\subsection{Un-Weighted Dataset Geometric Measure of Dispersion}

Consider a vectors $\mathrm{V}$ of data points $\mathrm{v}_{\mathrm{i}}$ such that all the points are not weighted or coefficient by any weights. A geometric measure of deviation from the mean $G$ for unweighted datasets will be given by the function;

$$
G=\left\{\begin{array}{cc}
\sqrt[n]{\prod_{i=1}^{p}\left|v_{i}-\bar{v}\right|} & \forall v_{i} \neq \bar{v} \\
0 & \forall v_{i}=\bar{v}
\end{array}\right.
$$

where $\mathrm{n}$ is the total number of data points in the dataset $\mathrm{V}$,

$\bar{v}$ is the mean for the dataset

$\mathrm{P}$ is the total number of data points which are not equal to the mean

$\left|v_{i}-\bar{v}\right|$ is the absolute deviation from the mean.

If all the data points $v_{i}=\bar{v}$ then the deviation from the mean will be zero, hence the mean deviation from the mean for such data points is hence zero, therefore, for data set with all points similar to each other the geometric deviation from the mean will be zero. For data set with at least $\mathrm{p}$ points not equal to the mean $\left(v_{i} \neq \bar{v}\right)$, the geometric mean deviation from the mean will be given by the formula;

$$
G=\sqrt[n]{\prod_{i=1}^{p}\left|v_{i}-\bar{v}\right|}
$$

To make the formula applicable for large data sets, a simplification of the formula can be carried out using the using the logarithmic transformation as below; 


$$
G=\left(\prod_{i=1}^{p}\left|v_{i}-\bar{v}\right|\right)^{\frac{1}{n}}
$$

Now let

$$
v_{i}-\bar{v}=d_{i}
$$

Then the function (5) can be written as;

$$
G=\left(\prod_{i=1}^{p}\left|d_{i}\right|\right)^{\frac{1}{n}}
$$

Now introducing natural logarithm on both sides we obtain the following function

$$
\ln (G)=\ln \left(\prod_{i=1}^{p}\left|d_{i}\right|\right)^{\frac{1}{n}}
$$

Equation (8) can be simplified as follows based on the logarithm rules;

$$
\ln (G)=\frac{1}{n} \ln \left(\prod_{i=1}^{p}\left|d_{i}\right|\right)
$$

Transforming back to get the original function by introducing exponential on both sides we obtain the following equation

$$
G=\left\{\begin{array}{cc}
\exp \left(\frac{1}{n} \ln \left(\prod_{i=1}^{n}\left|d_{i}\right|\right)\right. & \forall d_{i} \neq 0 \\
0 & \forall d_{i}=0
\end{array}\right.
$$

The function (9) will assist in reducing the problem that result from geometric rooting. The absolute value in the formula based on the algebraic theory helps in preventing us from having complex results when finding roots for negative numbers. The geometric mean is appropriate because it satisfies the algebraic formula [2];

$$
\prod_{i=1}^{n}\left|d_{i}\right|=\left|\prod_{i=1}^{n} d_{i}\right|
$$

Which is an improvement based on accuracy from the arithmetic averaging of absolute values [2];

$$
\frac{\sum_{i=1}^{n}\left|d_{i}\right|}{n}>\frac{\left|\sum_{i=1}^{n} d_{i}\right|}{n}
$$

Hence the geometric averaging gives more accurate results than arithmetic averaging. As a simplification and improvement, equation (9) can be simplified further by replacing the product with sum because of the logarithmic law which states that $\log$ of product is same as sum of individual logs hence;

$$
\ln (G)=\frac{1}{n} \sum_{i=1}^{p} \ln \left|d_{i}\right|
$$

Based on the transformation in (11) we can now rewrite (9) as follows;

$$
G=\left\{\begin{array}{cl}
\exp \left(\frac{1}{n} \sum_{i=1}^{p} \ln \left|d_{i}\right|\right) & \forall d_{i} \neq 0 \\
0 & \forall d_{i}=0
\end{array}\right.
$$

\subsection{Weighted Dataset Geometric Measure of Dispersion}

Consider a data vector $V$ such that $V=v_{1}, v_{2}, v_{3}, \ldots, v_{n}$ and a weighted vector $\zeta$ of the same dimension as $\mathrm{V}$, such that $\varsigma=\left[\varsigma_{1}, \varsigma_{2}, \varsigma_{3}, \ldots, \varsigma_{n}\right]$. The joint distribution of the two vectors results into a weighted vector of datasets $V \varsigma=\left[\varsigma_{1} v_{1}, \varsigma_{2} v_{2}, \varsigma_{3} v_{3}, \ldots \varsigma_{n} v_{n}\right]$. A geometric measure of deviation from the mean $G$ for weighted datasets will be given by the function;

$$
G=\left\{\begin{array}{cc}
\sum_{i=1}^{n} \varsigma_{i} \sqrt{\prod_{i=1}^{p}\left|v_{i}-\bar{v}\right|^{\varsigma_{i}}} & \forall v_{i} \neq \bar{v} \\
0 & \forall v_{i}=\bar{v}
\end{array}\right.
$$

where $\varsigma_{i}$ are the weights for every data point $v_{i}$.

$\bar{v}$ is the mean for the dataset

$\mathrm{P}$ is the total number of data points which are not equal to the mean

$\left|v_{i}-\bar{v}\right|$ is the absolute deviation from the mean.

If all the data points $v_{i}=\bar{v}$ then the deviation from the mean will be zero, hence the mean deviation from the mean for such data points is hence zero, therefore, for data set with all points similar to each other the geometric deviation from the mean will be zero. For data set with at least $\mathrm{p}$ points not equal to the mean $\left(v_{i} \neq \bar{v}\right)$, the geometric mean deviation from the mean will be given by the formula;

$$
G=\sum_{i=1}^{n} \varsigma_{i} \sqrt{\prod_{i=1}^{p}\left|v_{i}-\bar{v}\right|^{\varsigma_{i}}}
$$

To make the formula applicable for large data sets, a simplification of the formula can be carried out using the using the logarithmic transformation as below;

$$
G=\left(\prod_{i=1}^{p}\left|v_{i}-\bar{v}\right|^{\varsigma_{i}}\right)^{\frac{1}{\sum_{i=1}^{n} \varsigma_{i}}}
$$

Using (6) the function (14) can be written as; 


$$
G=\left(\prod_{i=1}^{p}\left|d_{i}\right|^{\varsigma_{i}}\right)^{\frac{1}{\sum_{i=1}^{n} \varsigma_{i}}}
$$

Now introducing natural logarithm on both sides we obtain the following function

$$
\ln (G)=\ln \left(\prod_{i=1}^{p}\left|d_{i}\right|^{\varsigma_{i}}\right)^{\frac{1}{\sum_{i=1}^{n} \varsigma_{i}}}
$$

Equation (16) can be simplified as follows based on the logarithm rules;

$$
\ln (G)=\frac{1}{\sum_{i=1}^{n} \varsigma_{i}} \ln \left(\prod_{i=1}^{p}\left|d_{i}\right|^{\varsigma_{i}}\right)
$$

Transforming back to get the original function by introducing exponential on both sides we obtain the following equation

$$
G=\left\{\begin{array}{cl}
\exp \left(\frac{1}{\sum_{i=1}^{n} \varsigma_{i}} \ln \left(\prod_{i=1}^{n}\left|d_{i}\right|^{\varsigma_{i}}\right)\right. & \forall d_{i} \neq 0 \\
0 & \forall d_{i}=0
\end{array}\right.
$$

The function (17) will assist in reducing the problem that result from geometric rooting. As a simplification and improvement, equation (17) can also be simplified further by replacing the product with sum based on logarithmic laws;

$$
\ln (G)=\frac{1}{\sum_{i=1}^{n} \varsigma_{i}} \sum_{i=1}^{p} \varsigma_{i} \ln \left|d_{i}\right|
$$

Based on the transformation in 18 we can now rewrite 17 as follows;

$$
G=\left\{\begin{array}{cc}
\exp \left(\frac{1}{\sum_{i=1}^{n} \varsigma_{i}} \sum_{i=1}^{p} \varsigma_{i} \ln \left|d_{i}\right|\right. \\
0 & \forall d_{i}=0
\end{array}\right.
$$

\subsection{Application on Probability Mass Functions}

Based on equation (18) it can be determined that $\ln (G)=E\left(\ln \left|d_{i}\right|\right)$ and that $\ln \left(d_{i}\right)$ is distributed with the same weights as $v_{i}$. Therefore, extending this relationship on probability mass functions. Assume that the variable $v_{i}$ is discrete with probability mass function $\varsigma\left(v_{i}\right)$ for all $i=1,2,3, \ldots, n$ and 0 otherwise. Assume that $\ln \left|d_{i}\right|$ which is equal to $\ln \left|v_{i}-\bar{v}\right|$ where $\bar{v}$ is the mean of the random variable $v$, is distributed in the same way as $v_{i}$ with a probability mass function $\varsigma\left(v_{i}\right)$. The geometric deviation for probability mass functions can be given as;

$$
G=\left\{\begin{array}{cl}
\exp \left(\sum_{i=1}^{n} \varsigma\left(v_{i}\right) \bullet \ln \left|d_{i}\right|\right) & \forall d_{i} \neq 0 \\
0 & \forall d_{i}=0
\end{array}\right.
$$

\subsection{Application on Probability Density Functions}

Extending the relationship in equation (18) on continuous random variables. Assume that the variable $v$ is continuous on the interval $a \leq v \leq b$ with probability density function $\varsigma(v)$. Assume that $\ln |d|$ which is equal to $\ln |v-\bar{v}|$ where $\bar{v}$ is the mean of the random variable $v$, is distributed in the same way as $v_{i}$ with a probability density function $\varsigma(v)$. The geometric deviation for probability density functions can be given as;

$$
G=\left\{\begin{array}{cl}
\exp \left(\int_{a}^{b} \varsigma(v) \cdot \ln |d| . d d\right) & \forall d \neq 0 \\
0 & \forall d=0
\end{array}\right.
$$

\section{Results}

\subsection{Application on Un-weighted Datasets}

Several simulations were conducted on both discrete and continuous data distributions of small populations of size 10 from Bernoulli, Binomial, Geometric, Normal, chi-square and F-distributed were. The results were as shown below;

\subsubsection{Bernoulli Population}

Consider a population of size 10 which is Bernoulli distributed with a probability of success 0.7 , the geometric measure of variation from the mean for the population estimated as illustrated in table 1;

The geometric measure of variation from the mean for unweighted dataset is given by the function;

$$
G=\left\{\begin{array}{cc}
\exp \left(\frac{1}{10} \sum_{i=1}^{p} \ln \left|d_{i}\right|\right) & \forall d_{i} \neq 0 \\
0 & \forall d_{i}=0
\end{array}\right.
$$


Table 1. Estimation for un-weighted Bernoulli population

\begin{tabular}{lllll}
\hline Population Number & Bernoulli Population & $d_{i}=x_{i}-0.8$ & $\left|d_{i}\right|$ & $\ln \left(\left|d_{i}\right|\right)$ \\
\hline 1 & 1 & 0.2 & 0.2 & -1.609437912 \\
2 & 1 & 0.2 & 0.2 & -1.609437912 \\
3 & 1 & 0.2 & 0.2 & -1.609437912 \\
4 & 1 & 0.2 & 0.2 & -1.609437912 \\
5 & 0 & -0.8 & 0.8 & -0.223143551 \\
6 & 1 & 0.2 & 0.2 & -1.609437912 \\
7 & 1 & 0.2 & 0.2 & -1.609437912 \\
8 & 1 & 0.2 & 0.2 & -1.609437912 \\
9 & 0 & -0.8 & 0.8 & -0.223143551 \\
10 & 1 & 0.2 & -1.609437912 \\
Mean & 0.8 & 0.2 & & -1.33217904 \\
\hline
\end{tabular}

Geometric measure $=\exp (-1.33217904)=0.263901582 \approx 0.2639$

\subsubsection{Binomial Population}

Consider a population of size 10 which is Binomial distributed with 30 trials and a probability of success 0.64 , the geometric measure of variation from the mean was estimated as shown in table 2.

Table 2. Estimation for un-weighted Binomial population

\begin{tabular}{lllll}
\hline Population Number & Binomial Population & $d_{i}=x_{i}-18.2$ & $\left|d_{i}\right|$ \\
\hline 1 & 18 & -0.2 & 0.2 & 1.2 \\
2 & 17 & -1.2 & 1.8 & -1.609437912 \\
3 & 20 & 1.8 & 0.2 & 0.182321557 \\
4 & 18 & -0.2 & 3.2 & -1.609437912 \\
5 & 15 & -3.2 & 2.2 & 1.16315081 \\
6 & 16 & -2.2 & 4.8 & 0.78845736 \\
7 & 23 & 4.8 & 1.2 & 1.568615918 \\
8 & 17 & --1.2 & 1.8 & 0.182321557 \\
9 & 20 & 1.8 & 0.2 & 0.587786665 \\
10 & 18 & -0.2 & & -1.609437912 \\
\hline
\end{tabular}

Geometric measure $=\exp (0.023212679)=1.02348419 \approx 1.0235$

\subsubsection{Geometric Population}

Consider a population of size 10 which is Geometric distributed with a probability of success 0.5 , the average deviation from the mean can be estimated using geometric measure of deviation from the mean for the population as illustrated in table 3;

Table 3. Estimation for un-weighted Geometric population.

\begin{tabular}{|c|c|c|c|c|}
\hline Population Number & Geometric Population & $d_{i}=x_{i}-1.9$ & $\left|d_{i}\right|$ & $\ln \left(\left|d_{i}\right|\right)$ \\
\hline 1 & 1 & -0.9 & 0.9 & -0.105360516 \\
\hline 2 & 1 & -0.9 & 0.9 & -0.105360516 \\
\hline 3 & 2 & 0.1 & 0.1 & -2.302585093 \\
\hline 4 & 3 & 1.1 & 1.1 & 0.09531018 \\
\hline 5 & 1 & -0.9 & 0.9 & -0.105360516 \\
\hline 6 & 1 & -0.9 & 0.9 & -0.105360516 \\
\hline 7 & 1 & -0.9 & 0.9 & -0.105360516 \\
\hline 8 & 3 & 1.1 & 1.1 & 0.09531018 \\
\hline 9 & 1 & -0.9 & 0.9 & -0.105360516 \\
\hline 10 & 5 & 3.1 & 3.1 & 1.131402111 \\
\hline Mean & 1.9 & & & -0.161272572 \\
\hline
\end{tabular}

Geometric measure $=\exp (-0.161272572)=0.851060065 \approx 0.8511$

\subsubsection{Chi-square Population}

Population of size 10 from Chi-square distribution with 1 degree of freedom was simulated as shown in table 4 . The calculation of geometric measure for the population was estimated as illustrated in table 4 . 
Table 4. Estimation for un-weighted Chi-square population.

\begin{tabular}{|c|c|c|c|c|}
\hline Population Number & Chi-square Population & $d_{i}=x_{i}-1.580066995$ & $\left|d_{i}\right|$ & $\ln \left(\left|d_{i}\right|\right)$ \\
\hline 1 & 3.217024949 & 1.636957954 & 1.636957954 & 0.492839614 \\
\hline 2 & 2.995690441 & 1.415623446 & 1.415623446 & 0.347570032 \\
\hline 4 & 1.481728301 & -0.098338694 & 0.098338694 & -2.319337698 \\
\hline 5 & 2.378023987 & 0.797956993 & 0.797956993 & -0.225700577 \\
\hline 6 & 0.189166369 & -1.390900625 & 1.390900625 & 0.329951469 \\
\hline 8 & 1.601235263 & 0.021168268 & 0.021168268 & -3.855251998 \\
\hline 9 & 0.180945899 & -1.399121096 & 1.399121096 & 0.335844251 \\
\hline 10 & 0.000163189 & -1.579903805 & 1.579903805 & 0.457363962 \\
\hline Mean & 1.580066995 & & & -0.330672829 \\
\hline
\end{tabular}

Geometric measure $=\exp (-0.330672829)=0.718440183 \approx 0.7184$

\subsubsection{Normal Distribution Population}

A population of size 10 from a Normal distribution with a mean of 10 and a standard deviation of 2 was simulated as illustrated in table 5. The estimation of average deviation from the mean using geometric measure of variation about the mean for the population was estimated as illustrated in table 5 .

Table 5. Estimation for un-weighted Normal population.

\begin{tabular}{lllll}
\hline Population Number & Normal Population & $d_{i}=x_{i}-9.560952744$ & $\left|d_{i}\right|$ & $\ln \left(\left|d_{i}\right|\right)$ \\
\hline 1 & 9.4732888 & -0.087663944 & 0.087663944 & -2.434244594 \\
2 & 10.0179588 & 0.457006053 & 0.457006053 & -0.783058643 \\
3 & 9.833743354 & 0.272790611 & 0.272790611 & -1.299050771 \\
4 & 9.843723505 & 0.282770761 & 0.282770761 & -1.263118741 \\
5 & 7.86179902 & -1.699153723 & 1.699153723 & 0.530130317 \\
6 & 10.54505528 & 0.98410254 & 0.98410254 & -0.01602518 \\
7 & 8.855622899 & -0.705329844 & 0.705329844 & -0.349089721 \\
8 & 9.473922934 & -0.087029809 & 0.087029809 & -2.441504582 \\
9 & 13.65741087 & 4.096458131 & 4.096458131 & 1.41012273 \\
10 & 6.047001969 & -3.513950775 & 3.513950775 & 1.256740981 \\
Mean & 9.560952744 & & & -0.53890982 \\
\hline
\end{tabular}

Geometric measure $=\exp (-0.53890982)=0.583383899 \approx 0.5834$

\subsubsection{F-distributed Population}

A population of size 10 from a F-distribution with 2 numerator and 5 denominator degrees of freedom was simulated as illustrated in table 6 . The calculation of geometric measure of variation about the mean for the population was calculated as illustrated in table 6 .

Table 6. Estimation for un-weighted Fisher's population.

\begin{tabular}{llll}
\hline Population Number & F-distribution Population & $d_{i}=x_{i}-2.273034666$ & $\left|d_{i}\right|$ \\
\hline 1 & 7.650678068 & 5.377643402 & 5.377643402 \\
2 & 2.329789864 & 0.056755198 & 0.056755198 \\
3 & 5.536239586 & 3.26320492 & -2.869008029 \\
4 & 1.738601369 & -0.534433297 & 3.26320492 \\
5 & 0.771963586 & -1.50107108 & 0.534433297 \\
6 & 0.555808899 & -1.717225767 & -0.626548351 \\
7 & 1.502359971 & -0.770674695 & 0.4061707108 \\
8 & 0.31742318 & -1.955611486 & 0.540717225767 \\
9 & 0.975575768 & -1.297458898 & 0.770674695 \\
10 & 1.351906368 & -0.921128298 & -0.260488921 \\
Mean & 2.273034666 & & 0.670702925 \\
\hline
\end{tabular}

Geometric measure $=\exp (0.090475837)=1.094695056 \approx 1.0947$

Based on the illustration shown by the calculations, the geometric measure can be used to estimate the average 
variation from the mean for both un-weighted discrete and continuous datasets.

\subsection{Application on Weighted Datasets}

The study intended to determine if the geometric measure could be used in the estimation of average deviations from the mean for weighted datasets with specific concentration on frequency distributions, where the frequencies were used as the weights of the respective data points. Simulated data for three discrete distributions (Bernoulli, Binomial and Geometric distributions) and three continuous distributions (Normal, Chisquare and Fishers/F-distributions) where used. 100 observations were simulated for each distribution after which the data were summarized into frequency distributions before geometric measure for the average deviation about the mean was estimated for each frequency distributions. The results of the simulation and the estimation was as illustrated below;

\subsubsection{Bernoulli Population}

Consider a population of size 100 which is Bernoulli distributed with a probability of success 0.7 , the frequency distribution and the estimation of the geometric measure variation from the mean for the population was as illustrated below;

The geometric measure of variation from the mean $(\mathrm{G})$ for weighted datasets is given by the function;

$$
G=\left\{\begin{array}{cc}
\exp \left(\frac{1}{\sum_{i=1}^{n} \varsigma_{i}} \sum_{i=1}^{p} \varsigma_{i} \ln \left(\left|d_{i}\right|\right)\right) & \forall d_{i} \neq 0 \\
0 & \forall d_{i}=0
\end{array}\right.
$$

Table 7. Estimation for weighted Bernoulli population.

\begin{tabular}{lllllll}
\hline Number $\left(v_{i}\right)$ & Frequency $\left(\varsigma_{i}\right)$ & $\varsigma_{i} v_{i}$ & $d_{i}=v_{i}-\bar{v}$ & $\left|v_{i}-\bar{v}\right|$ & $\ln \left|d_{i}\right|=\ln \left|v_{i}-\bar{v}\right|$ & $\varsigma_{i} \ln \left|d_{i}\right|=\varsigma_{i}\left|v_{i}-\bar{v}\right|$ \\
\hline 0 & 34 & 0 & -0.66 & 0.66 & -0.415515444 & -14.12752509 \\
1 & 66 & 66 & 0.34 & 0.34 & -1.078809661 & -71.20143765 \\
Total & 100 & 66 & & & & -85.32896275 \\
\hline
\end{tabular}

$$
\begin{gathered}
\operatorname{Mean}(\bar{v})=\frac{\sum_{i=1}^{n} \varsigma_{i} v_{i}}{\sum_{i=1}^{n} \varsigma_{i}}=\frac{66}{100}=0.66 \\
\ln (G)=\frac{\sum_{i=1}^{n} \varsigma_{i} \ln \left|d_{i}\right|}{\sum_{i=1}^{n} \varsigma_{i}}=\frac{-85.32896275}{100}=-0.8532896275
\end{gathered}
$$

Geometric measure $=\exp (-0.8532896275)=0.426011206 \approx 0.4260$

\subsubsection{Binomial Population}

Consider a population of size 100 which is Binomial distributed with 30 trials and a probability of success 0.64 , the frequency distribution and the estimation of the geometric measure variation from the mean for the population was as illustrated in table 8 .

Table 8. Estimation for weighted Binomial population.

\begin{tabular}{lllllll}
\hline Number $\left(v_{i}\right)$ & Frequency $\left(\varsigma_{i}\right)$ & $\varsigma_{i} v_{i}$ & $d_{i}=v_{i}-\bar{v}$ & $\left|v_{i}-\bar{v}\right|$ & $\ln \left|d_{i}\right|=\ln \left|v_{i}-\bar{v}\right|$ & $\varsigma_{i} \ln \left|d_{i}\right|=\varsigma_{i}\left|v_{i}-\bar{v}\right|$ \\
\hline 11 & 2 & 22.0 & -8.02 & 8.02 & 2.081938422 & 4.163876844 \\
12 & 1 & 12.0 & -7.02 & 7.02 & 1.948763218 & 1.948763218 \\
13 & 1 & 13.0 & -6.02 & 6.02 & 1.795087259 & 1.795087259 \\
14 & 3 & 42.0 & -5.02 & 5.02 & 1.613429934 & 4.840289801 \\
15 & 4 & 60.0 & -4.02 & 4.02 & 1.391281903 & 5.565127611 \\
16 & 5 & 80.0 & -3.02 & 3.02 & 1.105256831 & 5.526284157 \\
17 & 13 & 221.0 & -2.02 & 2.02 & 0.703097511 & 9.140267648 \\
18 & 7 & 126.0 & -1.02 & 1.02 & 0.019802627 & 0.138618391 \\
19 & 21 & 399.0 & -0.02 & 0.02 & -3.912023005 & -82.15248311 \\
20 & 9 & 180.0 & 0.98 & 0.98 & -0.020202707 & -0.181824366 \\
21 & 16 & 336.0 & 1.98 & 1.98 & 0.683096845 & 10.92954952 \\
22 & 8 & 176.0 & 2.98 & 2.98 & 1.091923301 & 8.735386404 \\
23 & 7 & 161.0 & 3.98 & 3.98 & 1.381281819 & 9.668972735 \\
\hline
\end{tabular}




\begin{tabular}{lllllll}
\hline Number $\left(v_{i}\right)$ & Frequency $\left(\varsigma_{i}\right)$ & $\varsigma_{i} v_{i}$ & $d_{i}=v_{i}-\bar{v}$ & $\left|v_{i}-\bar{v}\right|$ & $\ln \left|d_{i}\right|=\ln \left|v_{i}-\bar{v}\right|$ & $\varsigma_{i} \ln \left|d_{i}\right|=\varsigma_{i}\left|v_{i}-\bar{v}\right|$ \\
\hline 24 & 1 & 24.0 & 4.98 & 4.98 & 1.605429891 & 1.605429891 \\
25 & 2 & 50.0 & 5.98 & 5.98 & 1.788420568 & 3.576841136 \\
Total & 100 & 1902.0 & & & & -14.69981287 \\
\hline
\end{tabular}

$$
\begin{gathered}
\operatorname{Mean}(\bar{v})=\frac{\sum_{i=1}^{n} s_{i} v_{i}}{\sum_{i=1}^{n} \varsigma_{i}}=\frac{1902}{100}=19.02 \\
\ln (G)=\frac{\sum_{i=1}^{n} \varsigma_{i} \ln \left|d_{i}\right|}{\sum_{i=1}^{n} \varsigma_{i}}=\frac{-14.69981287}{100}=-0.1469981287
\end{gathered}
$$

Geometric measure $=\exp (-0.1469981287)=0.863295593 \approx 0.8633$

\subsubsection{Geometric Population}

Consider a population of size 100 which is Geometric distributed with a probability of success 0.5 , the frequency distribution and the estimation of the geometric measure variation from the mean for the population was as illustrated in table 9 .

Table 9. Estimation for weighted Geometric population.

\begin{tabular}{lllllll}
\hline Number $\left(v_{i}\right)$ & Frequency $\left(s_{i}\right)$ & $\varsigma_{i} v_{i}$ & $d_{i}=v_{i}-\bar{v}$ & $\left|v_{i}-\bar{v}\right|$ & $\ln \left|d_{i}\right|=\ln \left|v_{i}-\bar{v}\right|$ & $\varsigma_{i} \ln \left|d_{i}\right|=s_{i}\left|v_{i}-\bar{v}\right|$ \\
\hline 1 & 52 & 52 & -1.12 & 1.12 & 0.113328685 & 5.893091636 \\
2 & 22 & 44 & -0.12 & 0.12 & -2.120263536 & -46.6457978 \\
3 & 14 & 42 & 0.88 & 0.88 & -0.127833372 & -1.789667201 \\
4 & 4 & 16 & 1.88 & 1.88 & 0.631271777 & 2.525087107 \\
5 & 2 & 10 & 2.88 & 2.88 & 1.057790294 & 2.115580588 \\
6 & 2 & 12 & 3.88 & 3.88 & 1.355835154 & 2.711670307 \\
7 & 1 & 7 & 4.88 & 4.88 & 1.58514522 & 1.58514522 \\
8 & 1 & 8 & 5.88 & 5.88 & 1.771556762 & 1.771556762 \\
9 & 1 & 9 & 6.88 & 6.88 & 1.928618652 & 1.928618652 \\
12 & 1 & 12 & 9.88 & 9.88 & 2.290512512 & 2.290512512 \\
Total & 100 & 212.0 & & & & -27.61420221 \\
\hline
\end{tabular}

$$
\begin{gathered}
\operatorname{Mean}(\bar{v})=\frac{\sum_{i=1}^{n} \varsigma_{i} v_{i}}{\sum_{i=1}^{n} \varsigma_{i}}=\frac{212}{100}=2.12 \\
\ln (G)=\frac{\sum_{i=1}^{n} \varsigma_{i} \ln \left|d_{i}\right|}{\sum_{i=1}^{n} \varsigma_{i}}=\frac{-27.61420221}{100}=-0.2761420221
\end{gathered}
$$

Geometric measure $=\exp (-0.2761420221)=0.75870517 \approx 0.7587$

\subsubsection{Chi-square Population}

Population of size 100 from Chi-square distribution with 1 degree of freedom was simulated, the frequency distribution and the estimation of the geometric measure variation from the mean for the population was as illustrated in table 10 .

Table 10. Estimation for weighted Chi-square population.

\begin{tabular}{lllllll}
\hline Number $(v)$ & Midpoint $(v)$ & Frequency $(\varsigma)$ & $\boldsymbol{\zeta v}$ & $|\boldsymbol{d}|=|\boldsymbol{v}-\overline{\boldsymbol{v}}|$ & $\ln |\boldsymbol{d}|=\ln |\boldsymbol{v}-\overline{\boldsymbol{v}}|$ & $\boldsymbol{\zeta} \ln |\boldsymbol{d}|=\boldsymbol{\zeta} \ln |\boldsymbol{v}-\overline{\boldsymbol{v}}|$ \\
\hline $0-2$ & 1 & 82 & 82.0 & 0.5 & -0.69314718 & -56.83806881 \\
$2-4$ & 3 & 12 & 36.0 & 1.5 & 0.40546511 & 4.865581297 \\
$4-6$ & 5 & 5 & 25.0 & 3.5 & 1.25276297 & 6.263814842 \\
$6-8$ & 7 & 1 & 7.0 & 5.5 & 1.70474809 & 1.704748092 \\
Total & & 100 & 150.0 & & & -44.00392457 \\
\hline
\end{tabular}




$$
\begin{gathered}
\operatorname{Mean}(\bar{v})=\frac{\sum \varsigma v}{\sum \varsigma}=\frac{150}{100}=1.5 \\
\ln (G)=\frac{\sum \varsigma \ln |d|}{\sum \varsigma}=\frac{-44.00392457}{100}=-0.4400392457
\end{gathered}
$$

Geometric measure $=\exp (-0.4400392457)=0.644011146 \approx 0.6440$

\subsubsection{Normal Distribution Population}

A population of size 100 from a Normal distribution with a mean of 10 and a standard deviation of 2 was simulated. The frequency distribution and the estimation of the geometric measure variation from the mean for the population was as illustrated in table 11 .

Table 11. Estimation for weighted Normal Population.

\begin{tabular}{llllllll}
\hline Class & Midpoint $(v)$ & Frequency $(\varsigma)$ & $\varsigma v$ & $d=v-\bar{v}$ & $|d|=|v-\bar{v}|$ & $\ln |d|=\ln |v-\bar{v}|$ & $\varsigma \ln |d|=\varsigma \ln |v-\bar{v}|$ \\
\hline $4-6$ & 5 & 1 & 5.0 & -5.3 & 5.3 & 1.6677 & 1.667706821 \\
$6-8$ & 7 & 10 & 70.0 & -3.3 & 3.3 & 1.1939 & 11.93922468 \\
$8-10$ & 9 & 38 & 342.0 & -1.3 & 1.3 & 0.2624 & 9.96984205 \\
$10-12$ & 11 & 26 & 286.0 & 0.7 & 0.7 & -0.3567 & -9.273548542 \\
$12-14$ & 13 & 24 & 312.0 & 2.7 & 2.7 & 0.9933 & 23.83804255 \\
$14-16$ & 15 & 1 & 15.0 & 4.7 & 4.7 & 1.5476 & 1.547562509 \\
Total & 100 & 1030.0 & & & & 39.68883007 \\
\hline
\end{tabular}

$$
\begin{gathered}
\operatorname{Mean}(\bar{v})=\frac{\sum \varsigma v}{\sum \varsigma}=\frac{1030}{100}=10.3 \\
\ln (G)=\frac{\sum \varsigma \ln |d|}{\sum \varsigma}=\frac{39.68883007}{100}=0.3968883007
\end{gathered}
$$

Geometric measure $=\exp (0.3968883007)=1.487189803 \approx 1.4872$

\subsubsection{F-distributed Population}

A population of size 100 from a F-distribution with 2 numerator and 5 denominator degrees of freedom was simulated as illustrated in table 13. The calculation of geometric measure of variation about the mean for the population was calculated as illustrated in table 12 .

Table 12. Estimation for weighted Fisher's population.

\begin{tabular}{lllllll}
\hline Class & Midpoint $(v)$ & Frequency $(\varsigma)$ & $\varsigma v$ & $|d|=|v-\bar{v}|$ & $\ln |d|=\ln |v-\bar{v}|$ & $\varsigma \ln |d|=\varsigma \ln |v-\bar{v}|$ \\
\hline $0-2$ & 1 & 69 & 69.0 & 1.055 & 0.05354077 & 3.694312918 \\
$2-4$ & 3 & 18 & 54.0 & 0.945 & -0.05657035 & -1.018266327 \\
$4-6$ & 5 & 7 & 35.0 & 2.945 & 1.08010882 & 7.56076172 \\
$6-8$ & 7 & 5 & 35.0 & 4.945 & 1.59837697 & 7.991884825 \\
$8-10$ & 9 & 0 & 0.0 & 6.945 & 1.93802198 & 0 \\
$10-15$ & 12.5 & 1 & 12.5 & 10.445 & 2.34612340 & 2.346123395 \\
& Total & 100 & 205.5 & & & 20.57481653 \\
\hline
\end{tabular}

$$
\begin{gathered}
\operatorname{Mean}(\bar{v})=\frac{\sum \varsigma v}{\sum \varsigma}=\frac{205.5}{100}=2.055 \\
\ln (G)=\frac{\sum \varsigma \ln |d|}{\sum \varsigma}=\frac{20.57481653}{100}=0.2057481653
\end{gathered}
$$

Geometric measure $=\exp (0.0 .2057481653)=1.2284438 \approx 1.2284$

Based on the illustration shown by the calculations, the geometric measure can be used to estimate the average variation from the mean for both weighted discrete and continuous datasets. 


\subsection{Application on Probability Mass Functions}

The study considered various probability mass functions to illustrate how the function can be used in estimating the average variation from the mean for probability mass functions. The results were as illustrated below;

\subsubsection{Coin Tossing Gambling Game}

In a coin tossing gabling game, a player loses 1 shilling whenever a fair coin tosses head and gains 2 shillings whenever the fair coin tosses tail. Estimate the average variation of the gain using geometric measure of variation from the mean.

The geometric variation for the probability mass function is estimated using the functions;

$$
G=\left\{\begin{array}{cc}
\exp \left(\sum_{i=1}^{n} \varsigma\left(v_{i}\right) \bullet \ln \left|d_{i}\right|\right) & \forall d_{i} \neq 0 \\
0 & \forall d_{i}=0
\end{array}\right.
$$

The calculation of the estimate was as follows;

Table 13. Estimation for coin tossing gambling game.

\begin{tabular}{cccccc}
\hline Gain $\left(v_{i}\right)$ & Probability $\varsigma\left(v_{i}\right)$ & $v_{i} \varsigma\left(v_{i}\right)$ & $d_{i}$ & $\left|d_{i}\right|$ & \multicolumn{1}{c}{$\ln \left|d_{i}\right|$} \\
\hline-1 & 0.5 & -0.5 & -1.5 & 1.5 & 0.405465108 \\
2 & 0.5 & 1 & 1.5 & 1.5 & 0.202732554 \\
Total & 1 & 0.5 & 0.202732554 & 0.405465108 \\
\hline
\end{tabular}

$$
G=\exp (0.405465108)=1.5
$$

\subsubsection{Dice}

When an individual roll a die, he can get one of the numbers ranging from 1 to 6 , estimate the variation of obtaining a number when a die is rolled.

\begin{tabular}{|c|c|c|c|c|c|c|}
\hline Number $\left(v_{i}\right)$ & Probability $\varsigma\left(v_{i}\right)$ & $v_{i} \varsigma\left(v_{i}\right)$ & $d_{i}$ & $\left|d_{i}\right|$ & $\ln \left|d_{i}\right|$ & $\varsigma\left(v_{i}\right) \ln \left|d_{i}\right|$ \\
\hline 1 & 0.166666667 & 0.166666667 & -2.5 & 2.5 & 0.916290732 & 0.152715122 \\
\hline 2 & 0.166666667 & 0.333333333 & -1.5 & 1.5 & 0.405465108 & 0.067577518 \\
\hline 4 & 0.166666667 & 0.666666667 & 0.5 & 0.5 & -0.693147181 & -0.11552453 \\
\hline 5 & 0.166666667 & 0.833333333 & 1.5 & 1.5 & 0.405465108 & 0.067577518 \\
\hline 6 & 0.166666667 & 1 & 2.5 & 2.5 & 0.916290732 & 0.152715122 \\
\hline Total & & 3.5 & & & & 0.20953622 \\
\hline
\end{tabular}

Table 14. Estimation for rolling a dice.

$$
G=\exp (0.20953622)=1.233106037 \approx 1.2331
$$

\subsubsection{Bernoulli Distribution}

Consider a Bernoulli distribution with probability mass function;

$$
\varsigma(V)=\left\{\begin{array}{cc}
\varsigma^{v_{i}}(1-\varsigma)^{1-v_{i}} & V=0,1 \\
0 & \text { Otherwise }
\end{array}\right.
$$

Where $S$ is the probability of success, the natural logarithm of the geometric measure of variation about the mean can be estimated for the function by;

$$
\ln (G)=\sum_{i=0}^{1} \varsigma^{v_{i}}(1-\varsigma)^{1-v_{i}} \ln \left|v_{i}-\varsigma\right|
$$

Hence,

$$
G=\left\{\begin{array}{cl}
\exp \left(\sum_{i=0}^{1} \varsigma^{v_{i}}(1-\varsigma)^{1-v_{i}} \ln \left|v_{i}-\varsigma\right|\right) & \forall v_{i} \neq \varsigma \\
0 & \forall v_{i}=\varsigma
\end{array}\right.
$$

\subsubsection{Binomial Distribution}

Consider a binomial distribution with probability mass function

$$
\varsigma(V)=\left\{\begin{array}{cc}
\left(\begin{array}{c}
t \\
v_{i}
\end{array}\right) \varsigma^{v_{i}}(1-\varsigma)^{1-v_{i}} & V=0,1,2, \ldots, t \\
0 & \text { Otherwise }
\end{array}\right.
$$

Where $\zeta$ is the probability of success, the natural logarithm of the geometric measure of variation about the mean can be estimated for the function by;

$$
\ln (G)=\sum_{i=0}^{t}\left(\begin{array}{c}
t \\
v_{i}
\end{array}\right) \varsigma^{v_{i}}(1-\varsigma)^{1-v_{i}} \ln \left|v_{i}-t \varsigma\right|
$$


Hence,

$$
G=\left\{\begin{array}{cr}
\exp \left(\sum_{i=0}^{t}\left(\begin{array}{l}
t \\
v_{i}
\end{array}\right) \varsigma^{v_{i}}(1-\varsigma)^{1-v_{i}} \ln \left|v_{i}-t \zeta\right|\right) & \forall v_{i} \neq t \varsigma \\
0 & \forall v_{i}=t \varsigma
\end{array}\right.
$$

\subsubsection{Geometric Distribution}

Consider a geometric distribution with probability mass function

$$
\varsigma(V)=\left\{\begin{array}{cc}
\varsigma(1-\varsigma)^{v_{i}} & V=0,1,2, \ldots \\
0 & \text { Otherwise }
\end{array}\right.
$$

Where $\zeta$ is the probability of success, the natural logarithm of the geometric measure of variation about the mean can be estimated for the function by;

$$
\begin{aligned}
& \ln (G)=\sum_{i=0}^{\infty} \varsigma(1-\varsigma)^{v_{i}} \ln \left|v_{i}-\frac{1-\varsigma}{\varsigma}\right| \\
& \ln (G)=\sum_{i=0}^{\infty} \varsigma(1-\varsigma)^{v_{i}} \ln \left|\frac{\varsigma v_{i}-1+\varsigma}{\varsigma}\right| \\
& \ln (G)=\sum_{i=0}^{\infty} \varsigma(1-\varsigma)^{v_{i}} \ln \left|\frac{\varsigma v_{i}+\varsigma-1}{\varsigma}\right| \\
& \ln (G)=\sum_{i=0}^{\infty} \varsigma(1-\varsigma)^{v_{i}} \ln \left|\frac{\varsigma\left(v_{i}+1\right)-1}{\varsigma}\right|
\end{aligned}
$$

Hence,

$$
G=\left\{\begin{array}{cc}
\exp \left(\sum_{i=0}^{\infty} \varsigma(1-\varsigma)^{v_{i}} \ln \left|\frac{\zeta\left(v_{i}+1\right)-1}{\varsigma}\right|\right) & \forall v_{i} \neq \frac{1-\varsigma}{\varsigma} \\
0 & \forall v_{i}=\frac{1-\varsigma}{\varsigma}
\end{array}\right.
$$

\subsubsection{Poisson Distribution}

Consider a Poisson distribution with probability mass function;

$$
\varsigma(V)=\left\{\begin{array}{cc}
\frac{e^{-\varsigma} \varsigma^{v_{i}}}{v_{i} !} & V=0,1,2,3, \ldots \\
0 & \text { Otherwise }
\end{array}\right.
$$

Where $S$ is the average rate of event occurrence, the natural logarithm of the geometric measure of variation about the mean can be estimated for the function by;

$$
\ln (G)=\sum_{i=0}^{\infty} \frac{e^{-\varsigma} \varsigma^{v_{i}}}{v_{i} !} \ln \left|v_{i}-\varsigma\right|
$$

Hence,

$$
G=\left\{\begin{array}{cc}
\exp \left(\sum_{i=0}^{\infty} \frac{e^{-\varsigma} \varsigma^{v_{i}}}{v_{i} !} \ln \left|v_{i}-\varsigma\right|\right) & \forall v_{i} \neq \varsigma \\
0 & \forall v_{i}=\varsigma
\end{array}\right.
$$

The above illustrations show how the geometric measure about the mean can be used to estimate the average variations about the mean of probability mass functions.

\subsection{Application on Probability Density Functions}

The study considered various probability density functions to illustrate how the function can be used in estimating the average variation from the mean for probability density functions. The results were as illustrated below;

\subsubsection{Simple Probability Density Function}

Consider a random $v$ variable which is distributed in the interval $\left[\begin{array}{ll}0 & 1\end{array}\right]$ with a probability density function;

$$
\varsigma(v)=\left\{\begin{array}{lc}
1 & 0 \leq v \leq 1 \\
0 & \text { elsewhere }
\end{array}\right.
$$

We can estimate the average deviation from the mean for $v$ using the geometric measure as follows;

The geometric average deviation from the mean is given by the function;

$$
G=\left\{\begin{array}{cr}
\exp \left(\int_{a}^{b} \varsigma(v) \cdot \ln |v-\bar{v}| \cdot d v\right) & \forall v \neq \bar{v} \\
0 & \forall v=\bar{v}
\end{array}\right.
$$

Therefore, for the example pdf, we can obtain the expected value of the distribution as follows;

$$
E(V)=\int_{0}^{1} v \cdot d v=\frac{1}{2} v^{2}+\left.c\right|_{0} ^{1}=\left[\frac{1}{2}-0\right]=\frac{1}{2}
$$

The natural logarithm of the geometric measure of variation from the mean by definition is given by the formula;

$$
\ln (G)=E(\ln |v-\bar{v}|)=\int_{a}^{b} \varsigma(v) \cdot \ln |v-\bar{v}| \cdot d v
$$

Hence, for the above probability density function;

$$
\begin{aligned}
& \ln (G)=E\left(\ln \left|v-\frac{1}{2}\right|\right)=\int_{0}^{1} 1 \bullet \ln |v-\bar{v}| \cdot d v \\
& =1 \int_{0}^{1} \ln \left|v-\frac{1}{2}\right| d v=1\left[\left(v-\frac{1}{2}\right) \ln \left|v-\frac{1}{2}\right|-v+\frac{1}{2}\right]_{0}^{1} \\
& =1\left[\frac{1}{2} \ln \left(\frac{1}{2}\right)-1+\frac{1}{2}\right]-1\left[-\frac{1}{2} \ln \left(\frac{1}{2}\right)+\frac{1}{2}\right] \\
& =[-0.84657]-[0.84657] \\
& =-1.69315
\end{aligned}
$$

Therefore, the geometric measure of variation from the mean will be given by; 


$$
G=\exp (-1.69315)=0.18394
$$

\subsubsection{Uniform Distribution}

Consider a random variable $\mathrm{v}$ which is distributed uniformly in the interval $\left[\begin{array}{ll}2 & 10\end{array}\right]$. By definition, the probability density function of $\mathrm{v}$ will be given by;

$$
\varsigma(v)=\left\{\begin{array}{cc}
\frac{1}{10-2} & 2 \leq v \leq 10 \\
0 & \text { otherwise }
\end{array}\right.
$$

The expectation of the function is given by;

$$
E(v)=\frac{10+2}{2}=6
$$

Therefore, the natural logarithm of geometric measure of variation from the mean for the distribution will be given by;

$$
\begin{aligned}
& \ln (G)=E(\ln |v-6|)=\int_{2}^{10} \frac{1}{10-2} \cdot \ln |v-6| \cdot d v \\
& =\frac{1}{8} \int_{2}^{10} \ln |v-6| . d v=\frac{1}{8}[(v-6) \ln |v-6|-v+6]_{2}^{10} \\
& =\frac{1}{8}[(4 \ln (4)-10+6)-(-4 \ln (4)-2+6)] \\
& =\frac{1}{8}[1.54518+1.54518]=0.386294
\end{aligned}
$$

Therefore, the geometric measure of variation from the mean will be given by;

$$
G=\exp (0.386294)=1.471518 \approx 1.4715
$$

\subsubsection{Exponential Probability Density Function}

Consider an exponential distribution with probability density function;

$$
\varsigma(v)=\left\{\begin{array}{cc}
\varsigma e^{-\varsigma v} & v \geq 0 \\
0 & \text { otherwise }
\end{array}\right.
$$

By definition,

$$
E(v)=\frac{1}{\varsigma}
$$

Therefore, the natural logarithm of geometric measure about the mean is given by;

$$
\ln (G)=E\left(\ln \left|v-\frac{1}{\varsigma}\right|\right)=\int_{0}^{\infty} \varsigma e^{-\varsigma v} \cdot \ln \left|v-\frac{1}{\varsigma}\right| \cdot d v
$$

Hence,

$$
G=\left\{\begin{array}{cr}
\exp \left(\int_{0}^{\infty} \varsigma e^{-\varsigma v} \cdot \ln \left|v-\frac{1}{\varsigma}\right| \cdot d v\right) & \forall v \neq \frac{1}{\varsigma} \\
0 & \forall v=\frac{1}{\varsigma}
\end{array}\right.
$$

The above illustrations show that the geometric measure of variation from the mean can be used to estimate the average deviation from the mean, however, the function faces a challenge in the estimation especially when the limits are infinite. Also, the challenge arises when conducting partial integration for complex probability density functions which do not have finite integrals for the integration by parts. For example, consider the case of standard normal probability density function;

\subsubsection{Standard Normal}

Consider a standard normal distribution for a random variable v illustrated as follows;

$$
\zeta(v)=\left\{\begin{array}{cc}
\frac{1}{\sqrt{2 \pi}} e^{-\frac{v^{2}}{2}} & -\infty \leq v \leq \infty \\
0 & \text { otherwise }
\end{array}\right.
$$

Therefore, the natural logarithm of geometric measure about the mean is given by;

$$
\ln (G)=E(\ln |v|)=\frac{1}{\sqrt{2 \pi}} \int_{-\infty}^{\infty} e^{-\frac{v^{2}}{2}} \cdot \ln |v| \cdot d v
$$

Hence,

$$
G=\left\{\begin{array}{cl}
\exp \left(\frac{1}{\sqrt{2 \pi}} \int_{-\infty}^{\infty} e^{-\frac{v^{2}}{2}} \cdot \ln |v| . d v\right) & \forall v \neq 0 \\
0 & \forall v=0
\end{array}\right.
$$

This shows that it is almost impossible to integrate such functions. This is a short coming of the geometric measure of variation especially during its application on probability density functions

\section{Conclusion}

In conclusion, geometric measure of variation about the mean can be used to estimate the average deviation from the population mean for un-weighted datasets, weighted datasets, probability mass and probability density functions with finite intervals, however, the function faces serious integration problems when estimating the average deviation for probability density functions as a result of complexity in the integrations by parts involved and also integration on infinite intervals. The geometric measure of variation was also determined to give smaller estimates of the variation from the mean compared to standard deviation.

\section{References}

[1] Ahn, S., \& Fessler, J. A., (2003). Standard Errors of Mean, Variance, and Standard Deviation Estimators. EECS Department. The University of Michigan. U.S.A.

[2] Altman, D. G., \& Bland, J. M. (2005). Standard deviations and standard errors. BMJ Volume 331. 
[3] Bhardwaj, A., (2013). Comparative Study of Various Measures of Dispersion. Journal of Advances in Mathematics. Vol 1, No 1.

[4] Buckland, S. T., A. C. Studeny, A. E. Magurran, J. B. Illian, and S. E. Newson. (2011). The geometric mean of relative abundance indices: a biodiversity measure with a difference. Ecosphere 2 (9): 100. doi: 10.1890/ES11-00186.1.

[5] Clark, P. L., (2012). Number Theory: A Contemporary Introduction. Available http://math.uga.edu/ pete/4400FULL.pdf.

[6] Deshpande, S., Gogtay, N. J., Thatte, U. M., (2016). Measures of Central Tendency and Dispersion. Journal of the Association of Physicians of India. Vol. 64. July 2016.

[7] Grechuk, B., Molyboha, A., \& Zabarankin M., (2011). MeanDeviation Analysis in The Theory of Choice. Risk Analysis.

[8] Hu, S., (2010). Simple Mean, Weighted Mean, or Geometric Mean?. Presented at the 2010 ISPA/SCEA Joint Annual Conference and Training Workshop.

[9] Kum, S., \& Lim, Y., (2012). A Geometric Mean of Parameterized Arithmetic and Harmonic Means of Convex Functions. Hindawi Publishing Corporation. Volume 2012, Article ID 836804.

[10] Lawson, J. D., \& Lim, Y., (2001). The Geometric Mean, Matrices, Metrics, and More. The American Mathematical Monthly.

[11] Lee, D., In, J., \& Lee, S., (2015). Standard deviation and standard error of the mean. Korean journal of anesthesiology. 68. 220-3. 10.4097/kjae.2015.68.3.220.

[12] Leys, C., Klein, O., Bernard, P., \& Licata, L., (2013). Detecting outliers: Do not use standard deviation around the mean, use absolute deviation around the median. Journal of Experimental Social Psychology 49 (2013) 764-766.
[13] Manikandan, S., (2016). Measures of dispersion. Journal of Pharmacology and Pharmacotherapeutics. October-December 2011. Vol 2. Issue 4.

[14] McAlister, D., (1879). The Law of Geometric Mean. The Royal Society is collaborating with JSTOR to digitize, preserve, and extend access to Proceedings of the Royal Society of London.

[15] Mindlin, D., (2011). On the Relationship between Arithmetic and Geometric Returns. Cdi Advisors Research. LLC.

[16] Mohini, P. B., \&Prajakt, J. B., (2012). What to use to express the variability of data: Standard deviation or standard error of mean?. Perspectives in clinical research July 2012.

[17] Raymondo, J., (2015). Measures of Variation from Statistical Analysis in the Behavioral Sciences. Kendall Hunt Publishing.

[18] Roberson, Q. M., Sturman, M. C., \& Simons, T. L., (2007). Does the Measure of Dispersion Matter in Multilevel Research? A Comparison of the Relative Performance of Dispersion Indexes. Cornell University School of Hotel Administration. The Scholarly Commons.

[19] Roenfeldt, K., (2018). Better than average: Calculating Geometric Means Using SAS. Henry. M. Foundation for the Advancement of Military Medicine.

[20] Schuetter, J. (2007). Chapter 1. In J. Schuetter, measures of dispersation (pp. 45-54).

[21] Thenwall, M. (2018). The precision of the arithmetic mean, geometric mean and percentiles for citation data: An experimental simulation modelling approach. Statistical Cybermetrics Research Group, School of Mathematics and Computer Science, University of Wolverhampton, Wulfruna Street, Wolverhampton, UK. 